\title{
Contactless rheology of finite-size air-water interfaces
}

\author{
Vincent Bertin $\odot,{ }^{1,2,{ }^{*}}$ Zaicheng Zhang $\odot,{ }^{1,{ }^{*}}$ Rodolphe Boisgard, ${ }^{1}$ Christine Grauby-Heywang, ${ }^{1}$ Elie Raphaël $\odot,{ }^{2}$ \\ Thomas Salez $\odot{ }^{1,3, \dagger}$ and Abdelhamid Maali ${ }^{1}{ }^{1,+}$ \\ ${ }^{1}$ Univ. Bordeaux, CNRS, LOMA, UMR 5798, 33405 Talence, France \\ ${ }^{2}$ UMR CNRS Gulliver 7083, ESPCI Paris, PSL Research University, 75005 Paris, France \\ ${ }^{3}$ Global Station for Soft Matter, Global Institution for Collaborative Research and Education, \\ Hokkaido University, Sapporo, Hokkaido 060-0808, Japan
}

(Received 6 November 2020; revised 9 April 2021; accepted 16 June 2021; published 8 July 2021)

\begin{abstract}
We present contactless atomic force microscopy measurements of the hydrodynamic interactions between an oscillating sphere and a bubble in water at the microscale. The size of the bubble is found to have a significant effect on the response due to the long-range capillary deformation of the air-water interface. To rationalize the experimental data, we develop a viscocapillary lubrication model accounting for the finite-size effect. The comparison between experiments and theory allows us to measure the air-water surface tension, without contact and with the volume of liquid down to tens of microliters, paving the way toward robust contactless tensiometry of polluted air-water interfaces.
\end{abstract}

DOI: 10.1103/PhysRevResearch.3.L032007

The interface between two media has an energy cost per unit surface, called surface tension, resulting from the microscopic interactions of the constitutive molecules at the interface [1,2]. Surface tension is an important parameter in soft condensed matter and at small scales where capillary phenomena usually dominate. Examples include wetting properties [3,4], thin-film dynamics [5,6], and multiphase flows.

Surface active molecules, i.e., surfactants, are widely used to stabilize capillary interfaces on purpose, e.g., in emulsions or foams, but they are also inevitable due to pollution. These contaminants, which are usually adsorbed at the interface between two immiscible fluids, lower the surface tension and are responsible for specific rheological properties of the interface [7]. To understand the dynamics of soft materials, the interaction between objects such as droplets and bubbles, or to quantify the amount of interfacial contamination, capillary interfacial rheology is essential. Specifically, surface tension is measured by a large variety of techniques: the pendant-drop method [8], the spinning-drop method, and Wilhelmy plates or du Noüy rings [9], to name a few. Moreover, the interfacial rheology is usually measured with the Langmuir trough [10-12], oscillating-disk devices [13,14], particle tracking techniques [15-18], oscillating pendant drop [19-22], or through the measurement of capillary waves [23-27].

\footnotetext{
${ }^{*}$ These authors contributed equally to this paper.

†thomas.salez@u-bordeaux.fr

¥abdelhamid.maali@u-bordeaux.fr
}

Published by the American Physical Society under the terms of the Creative Commons Attribution 4.0 International license. Further distribution of this work must maintain attribution to the author(s) and the published article's title, journal citation, and DOI.
The atomic force microscope (AFM) is another powerful tool to probe capillary phenomena such as the interaction between bubbles [28,29] or droplets [30-32], as well as dynamical wetting [33-38] and the boundary conditions of flows at a water-air interface [39-41]. In a recent work, some of us used an AFM in dynamic mode in order to probe slippage at air-water interfaces containing impurities [40]. From the measurement of the hydrodynamic force, we have shown in particular that the viscous component of the force contains a signature of the advection of impurities adsorbed at the interface. Besides, the flow-induced advection of impurities at the interface also induces an elastic component in the force response. At small distances, the capillary deformation of the water-air interface becomes non-negligible and leads to an additional elastic component in the force response. The main objective of this previous work was to study the effect of impurity advection, and it was thus limited to large distances for which the capillary deformation of the bubble was negligible. Furthermore, this previous work focused only on interfaces with low contamination.

Contactless measurements using a dynamic AFM and a dynamic surface force apparatus (SFA) are increasingly used as a gentle probing method to quantify material properties [42-48]. Such measurements are based on an elastohydrodynamic (EHD) coupling that results from the hydrodynamic pressure which induces an elastic deformation of the substrate. The air-water interface is suitable to test such EHD coupling, since the interface deformation has an elasticlike capillary restoring response. It is described in the framework of the Young-Laplace equation that couples the hydrodynamic pressure and the capillary deformation of the interface. Importantly, the EHD coupling at the air-water interface has a long-range lateral extent due to the structure of the YoungLaplace equation. For large enough systems, the millimetric capillary length $\sqrt{\gamma /(\rho g)}$ characterizes the lateral extent of 


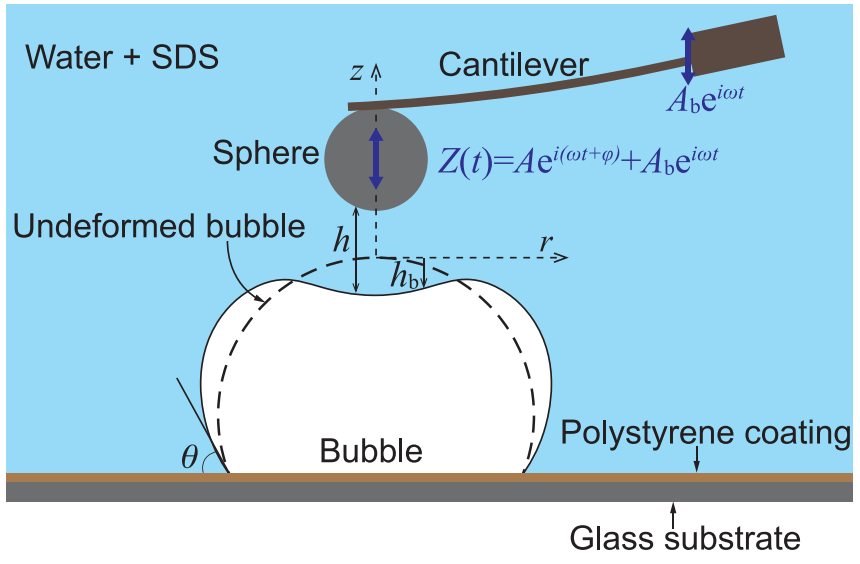

FIG. 1. A glass sphere attached to an AFM cantilever is driven to oscillate vertically near an air bubble deposited on a polystyrene surface in a SDS solution at a frequency of $\omega /(2 \pi) . \theta$ is the contact angle of the SDS solution on polystyrene in air. The vertical displacement of the sphere $Z(t)=A e^{i(\omega t+\varphi)}+A_{\mathrm{b}} e^{i \omega t}$ includes the cantilever deflection $A e^{i(\omega t+\varphi)}$ and the base displacement $A_{\mathrm{b}} e^{i \omega t}$. The motion results in an axisymmetric liquid-gap thickness profile $h(r, t)$, depending on the radial distance $r$ and time $t$, that includes an axisymmetric vertical profile $h_{\mathrm{b}}$ of the deformed bubble surface.

the capillary deformation, where $\gamma$ is the air-water surface tension, $\rho$ is the density of water, and $g$ is the gravity acceleration. However, for systems with lateral extents narrower than the capillary length, the deformation depends strongly on the system size [37]. Therefore, we expect the bubble stiffness defined as the ratio between the hydrodynamic force and bubble deformation to depend not only on the surface tension but also on the bubble size.

In this Letter, we present a contactless measurement of the rheological properties of air-water interfaces using dynamic AFM. The air-water interfaces are formed by depositing an air bubble in water with various concentrations of sodium dodecyl sulfate (SDS). The measured mechanical response presents two components: viscous and capillary, and both of them show a significant dependence on the bubble size. To rationalize the experimental results, we develop a model based on lubrication theory and the Young-Laplace equation, accounting for finite-size effects. From the comparison of the experimental results and theoretical predictions, the values of surface tension are extracted for different surfactant concentrations. As such, this method allows for robust probing of interfacial rheology in the absence of any direct contact.

The schematic of the experimental setup is shown in Fig. 1. The experimental method is described in [49] in detail. The cantilever is excited by the base oscillation $\mathcal{R}\left[A_{\mathrm{b}} e^{i \omega t}\right]$, where $\omega$ and $A_{\mathrm{b}}$ are the angular frequency and amplitude of the base vibration, respectively, $\mathcal{R}[$.$] denotes the real part, and$ $t$ denotes time. The system essentially behaves as a damped oscillator, where the vertical displacement $Z(t)$ of the center of mass of the sphere with respect to its rest position satisfies

$$
m_{\mathrm{c}} \ddot{Z}+\Gamma_{\text {bulk }} \dot{Z}+k_{\mathrm{c}} Z=F_{\mathrm{d}}+F,
$$

with $m_{\mathrm{c}}$ the effective mass (i.e., including the added fluid mass), $\Gamma_{\text {bulk }}$ the damping coefficient in the bulk, $k_{\mathrm{c}}$ the stiffness of the cantilever, $F_{\mathrm{d}}$ the driving force due to the imposed oscillation of the cantilever, and $F=\mathcal{R}\left[F^{*} e^{i \omega t}\right]$ the hydrodynamic force resulting from the interaction between the oscillating sphere and the air-water interface. The displacement $Z(t)$ of the sphere includes the cantilever deflection $\mathcal{R}\left[A e^{i(\omega t+\varphi)}\right]$ measured by AFM and the base displacement, and thus reads $Z(t)=\mathcal{R}\left[A e^{i(\omega t+\varphi)}+A_{\mathrm{b}} e^{i \omega t}\right]=$ $\mathcal{R}\left[Z^{*} e^{i \omega t}\right]$, where $A$ and $Z^{*}=A e^{i \varphi}+A_{\mathrm{b}}$ are real and complex amplitudes, respectively [49]. We further define the mechanical impedance $G^{*}=-F^{*} / Z^{*}$. Invoking the complex version of Eq. (1), the impedance reads

$$
G^{*}=-k_{\mathrm{c}}\left[1-\left(\frac{\omega}{\omega_{0}}\right)^{2}+i \frac{\omega}{\omega_{0} Q}\right] \frac{A e^{i \varphi}-A_{\infty} e^{i \varphi_{\infty}}}{A e^{i \varphi}+A_{\mathrm{b}}},
$$

where $A_{\infty}$ and $\varphi_{\infty}$ are, respectively, the amplitude $(A)$ and phase $(\varphi)$ measured far from the bubble (i.e., where $F$ vanishes), $\omega_{0}=\sqrt{k_{\mathrm{c}} / m_{\mathrm{c}}}$ is the bulk resonance frequency, and $Q=m_{\mathrm{c}} \omega_{0} / \Gamma_{\text {bulk }}$ is the bulk quality factor. Equation (2) provides a direct way to measure $G^{*}$ experimentally from the cantilever's deflection signal.

To model theoretically $G^{*}$, we consider the axisymmetric system composed of the rigid sphere located at an average distance $D$ from the apex of the undeformed air bubble. The ensemble is immersed in an incompressible Newtonian fluid with a dynamical shear viscosity $\eta$. We define the effective curvature radius $R_{\text {eff }}$ of the lubricated contact from $R_{\text {eff }}^{-1}=$ $R_{\mathrm{s}}^{-1}+R_{\mathrm{b}}^{-1}$, where $R_{\mathrm{s}}$ and $R_{\mathrm{b}}$ are the curvature radii of the sphere and bubble, respectively. We focus on the situation in which $D \ll R_{\text {eff }}$, so that we can invoke the lubrication approximation of the steady Stokes equations. The experiments are done at low enough frequencies so that we can assume a no-slip boundary condition at the air-water interface [40]. Such a condition is also assumed at the sphere-liquid interface. Therefore, the liquid-gap thickness obeys the Reynolds equation [50]:

$$
\frac{\partial h(r, t)}{\partial t}=\frac{1}{12 \eta r} \frac{\partial}{\partial r}\left[r h(r, t)^{3} \frac{\partial}{\partial r} p(r, t)\right],
$$

where $r$ is the radial coordinate, $p(r, t)$ is the excess hydrodynamic pressure field with respect to the rest state, $h(r, t)=$ $D+Z(t)+r^{2} /\left(2 R_{\mathrm{s}}\right)-h_{\mathrm{b}}(r, t)$ is the liquid-gap thickness, and $h_{\mathrm{b}}$ is the bubble surface profile (see Fig. 1). The latter follows the Young-Laplace equation:

$$
\frac{\gamma}{r} \frac{\partial}{\partial r}\left[r \frac{\frac{\partial h_{\mathrm{b}}}{\partial r}}{\sqrt{1+\left(\frac{\partial h_{\mathrm{b}}}{\partial r}\right)^{2}}}\right]=\Delta P(t)+p(r, t),
$$

where $\gamma$ denotes the air-water surface tension, and $\Delta P$ is the pressure drop across the interface. The contribution of Hamaker forces is neglected in the model, as the spherebubble distance in the experiment is in the $10 \mathrm{~nm}-20 \mu \mathrm{m}$ range, and thus typically larger than the distance below which these forces are dominant.

Even though the excess hydrodynamic pressure field is essentially localized near the apex of the bubble, over a radial extent that scales with the hydrodynamic radius $\sim \sqrt{2 R_{\text {eff }} D}$, the Young-Laplace equation induces deformations on larger scales-typically the millimetric capillary length [1]. Here, we consider bubbles with radii $R_{\mathrm{b}}$ smaller than the capillary length, so that (i) gravity is neglected, and (ii) the 
radial extent of the bubble's deformation induced by the hydrodynamic pressure is rather set by $R_{\mathrm{b}}$. Thus, we expect finite-size effects. Interestingly, there is still a natural scale separation since $\sqrt{2 R_{\mathrm{eff}} D} \ll R_{\mathrm{b}}$ in practice. We thus use an asymptotic-matching method to solve the problem [31]. The outer solution (denoted with the subscript "out") is solved exactly and depends directly on the hydrodynamic force $F(t)=$ $2 \pi \int_{0}^{\infty} d r r p(r, t)[51]$. We treat the inner hydrodynamic region (denoted with the subscript "in") as a boundary layer of the full interfacial deformation. We further assume a small amplitude of the sphere's oscillation, and we expand the inner surface profile of the bubble as $h_{\mathrm{b}, \text { in }}(r, t)=-r^{2} /\left(2 R_{\mathrm{b}}\right)-$ $u_{\text {in }}(r, t)$, where $u_{\text {in }}$ is the inner flow-induced capillary deformation of the air-water interface. The linearization of Eq. (4) near the apex of the bubble, combined with the air-volume conservation, yields [51]

$$
-\frac{\gamma}{r} \frac{\partial}{\partial r}\left(r \frac{\partial u_{\mathrm{in}}}{\partial r}\right)=-\frac{F(t)}{\pi R_{\mathrm{b}}^{2}} \frac{1}{1+\cos \theta}+p(r, t),
$$

where $\theta$ is the contact angle (see Fig. 1), and where we assumed the contact line to be pinned. The matching of the inner and the outer solutions imposes the asymptotic behavior $u_{\text {in }}(r, t) \sim-\frac{F(t)}{2 \pi \gamma}\left[1-\ln \left(\frac{1+\cos \theta}{1-\cos \theta}\right)+\ln \left(\frac{r}{2 R_{\mathrm{b}}}\right)\right]$ for $r \gg$ $\sqrt{2 R_{\text {eff }} D}$. We invoke the linear-response framework, and we write $u_{\text {in }}(r, t)=\mathcal{R}\left[u_{\mathrm{in}}^{*}(r) e^{i \omega t}\right]$ and $p(r, t)=\mathcal{R}\left[p^{*}(r) e^{i \omega t}\right]$, with $u_{\mathrm{in}}^{*}(r)$ and $p^{*}(r)$ the corresponding complex amplitudes. Finally, the amplitude of the hydrodynamic force reads $F^{*}=$ $2 \pi \int_{0}^{\infty} d r r p^{*}(r)$ in the lubrication approximation, which allows us to compute the mechanical impedance $G^{*}$ [51].

The experiments are performed using an AFM (Bioscope, Bruker) equipped with a liquid cell (DTFML-DD-HE). A spherical borosilicate particle (MO-Sci Corporation) with an $R_{\mathrm{S}}=54 \pm 2 \mu \mathrm{m}$ radius is glued at the edge of a silicon nitride cantilever (ORC8-10, Bruker AFM Probes). The stiffness $k_{\mathrm{c}}=0.20 \pm 0.01 \mathrm{~N} / \mathrm{m}$ of the cantilever (with the sphere attached to it) is determined from the drainage method [52]. The bulk resonance frequency $\omega_{0} /(2 \pi)=1240 \pm 3 \mathrm{~Hz}$ and the bulk quality factor $Q=3.4 \pm 0.1$ are obtained from the resonance spectrum at a large distance [49]. Air microbubbles are deposited onto spin-coated polystyrene layers, within SDS solutions in water. The SDS concentrations $C$ are in the $0.2-$ $40 \mathrm{mM}$ range. As measured with an optical microscope, the bubble radii $R_{\mathrm{b}}$ are in the $0.2-0.6 \mathrm{~mm}$ range, and the contact angles $\theta$ (see the definition in Fig. 1) are in the $40^{\circ}-90^{\circ}$ range, with the exact value depending on $C$. A multiaxis piezo stage (NanoT series, Mad City Labs) is used to control the distance between the sphere and the bubble by imposing a displacement to the substrate at very low velocity. The amplitude $A$ and phase $\varphi$ of the cantilever's deflection signal are measured by a lock-in amplifier (Model 7280, Signal Recovery), and they are recorded versus the piezo displacement. Additionally, the dc component of the cantilever's deflection is also recorded and used to determine the average gap distance $D$. We stress that the amplitude of the spherical probe's oscillation is a few nanometers, and always less than $3.5 \mathrm{~nm}$, which is itself smaller than $D$, fixed to be in the $10 \mathrm{~nm}-20 \mu \mathrm{m}$ range.

The real and imaginary parts of the measured mechanical impedance $G^{*}=G^{\prime}+i G^{\prime \prime}$ are plotted in Fig. 2 as functions of the average sphere-bubble distance $D$ for two frequencies

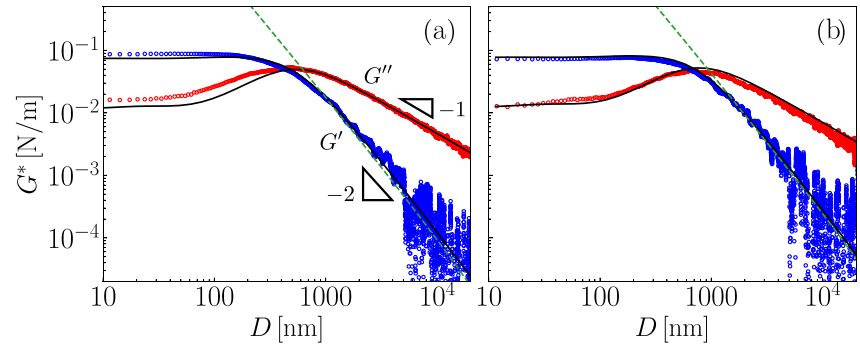

FIG. 2. Real (blue circles) and imaginary (red circles) parts of the measured mechanical impedance $G^{*}=G^{\prime}+i G^{\prime \prime}$ vs average spherebubble distance $D$ for a surfactant concentration $C=1 \mathrm{mM}$, and frequencies $\omega /(2 \pi)=200 \mathrm{~Hz}$ (a) and $300 \mathrm{~Hz}$ (b). The bubble radius is $R_{\mathrm{b}}=346 \pm 2 \mu \mathrm{m}$ and the contact angle is $\theta=81^{\circ} \pm 2^{\circ}$. The best fits to the model [51] are displayed with solid black lines, using a single fitting parameter $\gamma=54 \pm 4 \mathrm{mN} / \mathrm{m}$. The large-distance asymptotic solution for $G^{\prime}$ [see Eq. (6)] is also shown with green dashed lines. The slope triangles indicate power-law exponents.

and a given surfactant concentration. Best fits to the model [51] are also shown, in good agreement with the data, the air-water surface tension $\gamma=54 \pm 4 \mathrm{mN} / \mathrm{m}$ being the only fitting parameter. Furthermore, two asymptotic regimes can be observed, at large and small distance, respectively. They cross over near $D \approx 1000 \mathrm{~nm}$, which corresponds to the typical viscocapillary distance $D_{\mathrm{c}}=16 R_{\text {eff }}^{2} \eta \omega / \gamma$ emerging from the model [51], and equal to 771 and $1160 \mathrm{~nm}$ in Figs. 2(a) and 2(b), respectively. At large distance, the viscous contribution $G^{\prime \prime}$ dominates and follows a $\sim D^{-1}$ scaling law, as expected from the asymptotic expression $G^{\prime \prime} \simeq 6 \pi \eta R_{\text {eff }}^{2} \omega / D$ [43]. In contrast, the restoring contribution $G^{\prime}$ due to the air-water capillary interface appears with an apparent $\sim D^{-2}$ scaling law at large distance. We stress that the latter is not an exact scaling law, due to a logarithmic correction [51]:

$$
\begin{aligned}
G^{\prime}(D) \simeq & \frac{9 \pi \eta^{2} R_{\mathrm{eff}}^{4} \omega^{2}}{\gamma D^{2}}\left[-3+\ln (4)-2 \ln \left(\frac{1+\cos \theta}{1-\cos \theta}\right)\right. \\
& \left.+\ln \left(\frac{R_{\mathrm{b}}^{2}}{2 R_{\mathrm{eff}} D}\right)\right] .
\end{aligned}
$$

At small distance, both $G^{\prime}$ and $G^{\prime \prime}$ saturate to constant values, which is reminiscent of elastohydrodynamic responses near soft substrates [43-45,48,53,54], and might be related to saturations in the deformation and pressure fields. At such small distances, the capillary deformation of the bubble surface essentially accommodates the sphere's oscillation, and the liquid is no longer expelled from the gap, which further leads to a stronger capillary response than the viscous one.

To reveal the importance of finite-size effects in the viscocapillary response, we introduce the dimensionless mechanical impedance $\mathcal{G}^{*}=G^{*} D_{\mathrm{c}} /\left(6 \pi \eta \omega R_{\text {eff }}^{2}\right)$. In Fig. 3, the experimental and theoretical dimensionless mechanical impedances are plotted versus the dimensionless average sphere-bubble distance for three bubble radii. Except for the viscous contribution in the large-distance limit, the dimensionless impedance is generally found to depend on the bubble size in a nontrivial way, which is correctly reproduced by the model. This observation highlights the importance of finite-size effects in viscocapillary interactions, resulting 


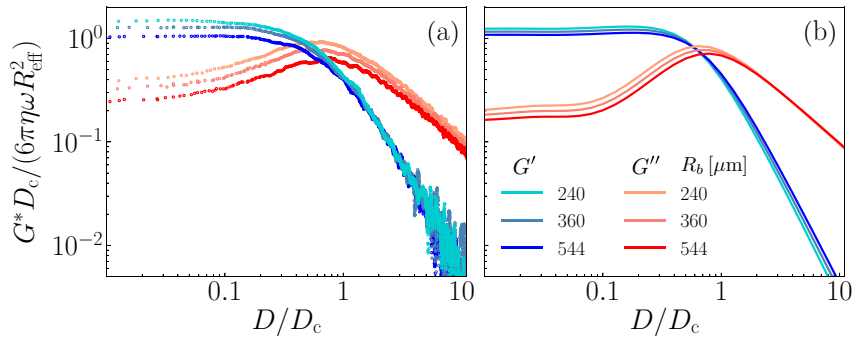

FIG. 3. Dimensionless mechanical impedance vs dimensionless distance, for three bubble sizes as indicated, a single frequency $\omega /(2 \pi)=200 \mathrm{~Hz}$, and a single surfactant concentration $C=1 \mathrm{mM}$. The experimental data are shown in (a). The results of the model are plotted in (b), using the previously obtained best-fit parameter $\gamma=54 \mathrm{mN} / \mathrm{m}$.

from the long-range capillary deformation of the air-water interface. We note that the logarithmic correction in the largedistance asymptotic expression of the capillary contribution [see Eq. (6)] contains a bubble-size dependence that cannot be resolved with the AFM sensitivity and the current bubblesize range. At small distance, the size dependence is more pronounced and both the real and imaginary parts of the dimensionless impedance decrease when increasing the bubble size.

Having discussed the finite-size effects on the global hydrodynamic force, we now investigate their influence on the amplitudes of the local excess pressure and deformation fields. To do so, we perform numerical integrations of Eqs. (3) and (5) using the asymptotic expression for the inner deformation field [51]. Figure 4 shows the results for $D / D_{\mathrm{c}}=0.3$, with the same parameters as in Fig. 3. We observe that the real and imaginary parts of the dimensionless amplitude of the excess pressure field decay rapidly on a typical distance $\sim \sqrt{R_{\text {eff }} D}$, and they depend weakly on the bubble radius. In sharp contrast, the real and imaginary parts of the dimensionless amplitude of the inner deformation field largely depend
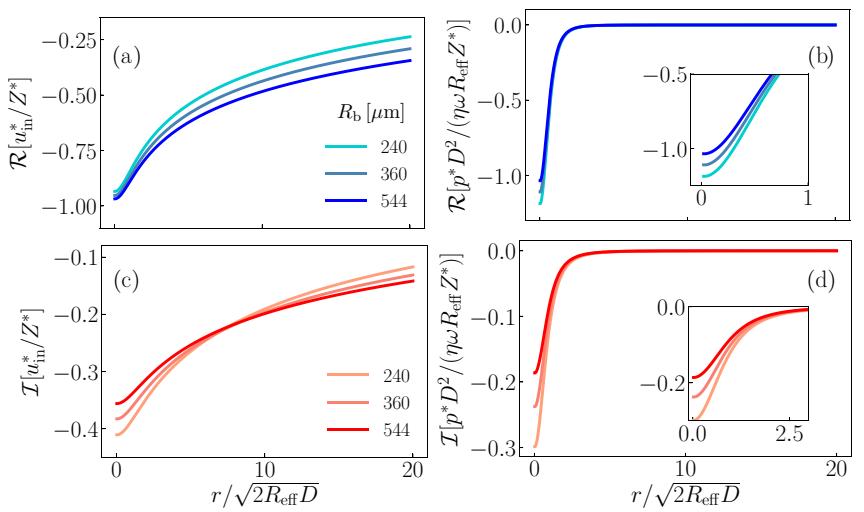

FIG. 4. Real (a) and imaginary (c) parts of the amplitude of the dimensionless inner deformation field as functions of the dimensionless radial coordinate, at a dimensionless distance $D / D_{\mathrm{c}}=0.3$, for the three bubble radii of Fig. 3, as obtained from the model [51]. Similarly, the real and imaginary parts of the amplitude of the dimensionless excess pressure field are plotted in panels (b) and (d), respectively. The insets display zooms near the symmetry axis.

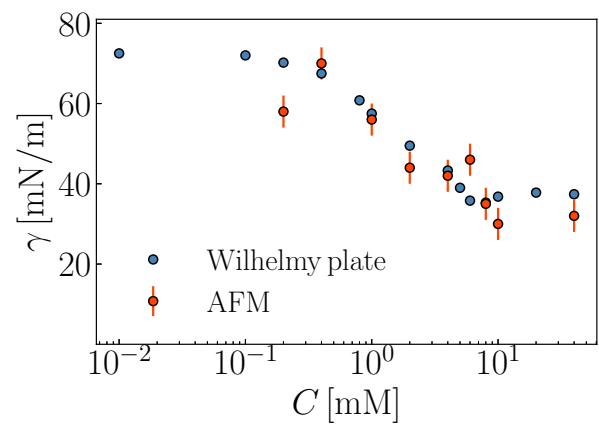

FIG. 5. Air-water surface tension as a function of surfactant (SDS) concentration, as obtained from fits (see Fig. 2) of the AFM experimental data by the model (red dots). Statistical error bars associated with multiple measurements at different frequencies are indicated. For comparison, independent measurements using the Wilhelmy-plate method are provided (blue dots).

on the bubble radius, as well as on the contact angle (not shown).

So far, the air-water surface tension was considered as a free parameter and was fixed by fitting the AFM experimental data to the model. The fitted values of the surface tension as a function of the SDS concentration in water are shown in Fig. 5. We observe that the surface tension globally decreases with increasing surfactant concentration, as expected. At surfactant concentrations smaller than $\sim 0.5 \mathrm{mM}$, the surface tension is close to the $72 \mathrm{mN} / \mathrm{m}$ value for pure water. At concentrations larger than $\sim 8 \mathrm{mM}$, the surface tension saturates to a value on the order of $30 \mathrm{mN} / \mathrm{m}$. The critical micellar concentration of SDS in water is estimated to be around $8 \mathrm{mM}$ $[55,56]$, which is in agreement with the latter observation. The uncertainty on the fitted values of the surface tension is on the order of $\pm 4 \mathrm{mN} / \mathrm{m}$, which mainly results from the fact that the experiments at different frequencies lead to slight variations.

Finally, we discuss the capacity of our method to be used as a robust tensiometer. To do so, we perform independent tensiometry experiments on similar air-water-SDS interfaces using the Wilhelmy-plate method [9]. The results are shown in Fig. 5, and they agree well with the ones obtained with our method. Possible systematic deviations at the highest concentrations may result from a surfactant-induced depinning of the contact line of the bubble on the substrate [57]. In such a scenario, the hydrodynamic pressure would not only trigger a local capillary deformation [see Eq. (4)], but would also induce a spreading-dewetting cycle of the bubble on the substrate. In addition, the bubble resonance frequency being lower at lower surface tension, capillary waves might be excited at the air-water interface at large surfactant concentrations. Besides, at the smallest concentration $(0.2 \mathrm{mM})$ used in the AFM experiment, the air-water interface may not be entirely covered with an adsorbed surfactant layer, potentially resulting in slippage. In such a scenario, the surfactant advection induced by the flow would add an elastic component to the mechanical response $[39,40]$, which might explain the small deviation observed in Fig. 5.

In conclusion, we have studied the viscocapillary interaction between an air bubble and a spherical probe attached 
to an AFM cantilever, and immersed within a surfactant solution in water. The sphere was oscillated in the direction normal to the air-water interface, thus generating a flow and an associated hydrodynamic pressure field that could deform the interface. The resulting force exerted on the sphere was measured as a function of the sphere-bubble distance, and it was found to depend on the bubble size. We also developed a model, coupling axisymmetric lubrication flow and capillary deformations, and accounting for finite-size effects through an asymptotic-matching method. The experimental results were found to be in good agreement with the model, the air-water surface tension being the single free parameter. Finally, from a comparison with independent tensiometry measurements using the Wilhelmy-plate method, we discussed the capacity of our method to measure surface tensions robustly. The volume of the liquid required in our method can be as small as tens of microliters. Altogether, this work paves the way to contactless capillary rheology, with fundamental perspectives in confined soft matter, and practical applications toward micromonitoring of water contamination.

The authors thank Elisabeth Charlaix for preliminary discussions, as well as Samir Almohamad for technical assistance on the Wilhelmy-plate calibration experiments. The authors acknowledge financial support from Agence Nationale de la Recherche (Grants No. ANR-19-CE30-0012 and No. ANR-21-ERCC-0010-01 EMetBrown). Z.Z. acknowledges financial support from the China Scholarship Council.
[1] P.-G. De Gennes, F. Brochard-Wyart, and D. Quéré, Capillarity and Wetting Phenomena: Drops, Bubbles, Pearls, Waves (Springer Science \& Business Media, New York, 2013).

[2] A. Marchand, J. H. Weijs, J. H. Snoeijer, and B. Andreotti, Why is surface tension a force parallel to the interface? Am. J. Phys. 79, 999 (2011).

[3] P.-G. De Gennes, Wetting: statics and dynamics, Rev. Mod. Phys. 57, 827 (1985).

[4] D. Bonn, J. Eggers, J. Indekeu, J. Meunier, and E. Rolley, Wetting and spreading, Rev. Mod. Phys. 81, 739 (2009).

[5] A. Oron, S. H. Davis, and S. G. Bankoff, Long-scale evolution of thin liquid films, Rev. Mod. Phys. 69, 931 (1997).

[6] R. V. Craster and O. K. Matar, Dynamics and stability of thin liquid films, Rev. Mod. Phys. 81, 1131 (2009).

[7] D. Langevin, Rheology of adsorbed surfactant monolayers at fluid surfaces, Annu. Rev. Fluid Mech. 46, 47 (2014).

[8] J. D. Berry, M. J. Neeson, R. R. Dagastine, D. Y. Chan, and R. F. Tabor, Measurement of surface and interfacial tension using pendant drop tensiometry, J. Colloid Interface Sci. 454, 226 (2015).

[9] J. Drelich, C. Fang, and C. White, Measurement of interfacial tension in fluid-fluid systems, in Encyclopedia of Surface and Colloid Science, edited by A. T. Hubbard (Marcel Dekker, Inc., New York, 2002), pp. 3152-3166.

[10] G. L. Gaines, Insoluble Monolayers at Liquid-gas Interfaces (Interscience, New York, 1966).

[11] S. Reynaert, C. F. Brooks, P. Moldenaers, J. Vermant, and G. G. Fuller, Analysis of the magnetic rod interfacial stress rheometer, J. Rheol. 52, 261 (2008).

[12] D. K. Schwartz, C. M. Knobler, and R. Bruinsma, Direct Observation of Langmuir Monolayer Flow Through a Channel, Phys. Rev. Lett. 73, 2841 (1994).

[13] R. Miller, V. B. Fainerman, J. Krägel, and G. Loglio, Surface rheology of adsorbed surfactants and proteins, Curr. Opin. Colloid Interface Sci. 2, 578 (1997).

[14] P. Erni, P. Fischer, E. J. Windhab, V. Kusnezov, H. Stettin, and J. Läuger, Stress- and strain-controlled measurements of interfacial shear viscosity and viscoelasticity at liquid/liquid and gas/liquid interfaces, Rev. Sci. Instrum. 74, 4916 (2003).

[15] F. Ortega, H. Ritacco, and R. G. Rubio, Interfacial microrheology: particle tracking and related tech- niques, Curr. Opin. Colloid Interface Sci. 15, 237 (2010).

[16] J. R. Samaniuk and J. Vermant, Micro and macrorheology at fluid-fluid interfaces, Soft matter 10, 7023 (2014).

[17] M. H. Lee, C. P. Lapointe, D. H. Reich, K. J. Stebe, and R. L. Leheny, Interfacial hydrodynamic drag on nanowires embedded in thin oil films and protein layers, Langmuir 25, 7976 (2009).

[18] V. Bergeron and D. Langevin, Monolayer Spreading of Polydimethylsiloxane Oil on Surfactant Solutions, Phys. Rev. Lett. 76, 3152 (1996).

[19] A. Yeung, T. Dabros, and J. Masliyah, Dissipative interfaces and departures from the young- laplace equation, Langmuir $\mathbf{1 3}$ 6597 (1997).

[20] M. Leser, S. Acquistapace, A. Cagna, A. Makievski, and R. Miller, Limits of oscillation frequencies in drop and bubble shape tensiometry, Colloids Surf., A 261, 25 (2005).

[21] F. Ravera, G. Loglio, and V. I. Kovalchuk, Interfacial dilational rheology by oscillating bubble/drop methods, Curr. Opin. Colloid Interface Sci. 15, 217 (2010).

[22] A. Yeung and L. Zhang, Shear effects in interfacial rheology and their implications on oscillating pendant drop experiments, Langmuir 22, 693 (2006).

[23] D. Langevin et al., Light Scattering by Liquid Surfaces and Complementary Techniques (Dekker, New York, 1992).

[24] J. C. Earnshaw, Surface light scattering: a methodological review, Appl. Opt. 36, 7583 (1997).

[25] R. Miller, J. K. Ferri, A. Javadi, J. Krägel, N. Mucic, and R. Wüstneck, Rheology of interfacial layers, Colloid Polym. Sci. 288, 937 (2010).

[26] A. Shmyrov, A. Mizev, A. Shmyrova, and I. Mizeva, Capillary wave method: An alternative approach to wave excitation and to wave profile reconstruction, Phys. Fluids 31, 012101 (2019).

[27] F. Monroy and D. Langevin, Direct Experimental Observation of the Crossover from Capillary to Elastic Surface Waves on Soft Gels, Phys. Rev. Lett. 81, 3167 (1998).

[28] I. U. Vakarelski, J. Lee, R. R. Dagastine, D. Y. Chan, G. W. Stevens, and F. Grieser, Bubble colloidal afm probes formed from ultrasonically generated bubbles, Langmuir 24, 603 (2008).

[29] I. U. Vakarelski, R. Manica, X. Tang, S. J. O’Shea, G. W. Stevens, F. Grieser, R. R. Dagastine, and D. Y. Chan, Dynamic 
interactions between microbubbles in water, Proc. Natl. Acad. Sci. (USA) 107, 11177 (2010).

[30] R. R. Dagastine, R. Manica, S. L. Carnie, D. Chan, G. W. Stevens, and F. Grieser, Dynamic forces between two deformable oil droplets in water, Science 313, 210 (2006).

[31] D. Y. Chan, E. Klaseboer, and R. Manica, Film drainage and coalescence between deformable drops and bubbles, Soft Matter 7, 2235 (2011).

[32] R. F. Tabor, F. Grieser, R. R. Dagastine, and D. Y. Chan, Measurement and analysis of forces in bubble and droplet systems using afm, J. Colloid Interface Sci. 371, 1 (2012).

[33] S. Ecke, M. Preuss, and H.-J. Butt, Microsphere tensiometry to measure advancing and receding contact angles on individual particles, J. Adhes. Sci. Technol. 13, 1181 (1999).

[34] X. Xiong, S. Guo, Z. Xu, P. Sheng, and P. Tong, Development of an atomic-force-microscope-based hanging-fiber rheometer for interfacial microrheology, Phys. Rev. E 80, 061604 (2009).

[35] M. Delmas, M. Monthioux, and T. Ondarçuhu, Contact Angle Hysteresis at the Nanometer Scale, Phys. Rev. Lett. 106, 136102 (2011).

[36] S. Guo, M. Gao, X. Xiong, Y. J. Wang, X. Wang, P. Sheng, and P. Tong, Direct Measurement of Friction of a Fluctuating Contact Line, Phys. Rev. Lett. 111, 026101 (2013).

[37] J. Dupré de Baubigny, M. Benzaquen, L. Fabié, M. Delmas, J.-P. Aimé, M. Legros, and T. Ondarçuhu, Shape and effective spring constant of liquid interfaces probed at the nanometer scale: Finite size effects, Langmuir 31, 9790 (2015).

[38] C. Mortagne, K. Lippera, P. Tordjeman, M. Benzaquen, and T. Ondarçuhu, Dynamics of anchored oscillating nanomenisci, Phys. Rev. Fluids 2, 102201(R) (2017).

[39] O. Manor, I. U. Vakarelski, G. W. Stevens, F. Grieser, R. R. Dagastine, and D. Y. Chan, Dynamic forces between bubbles and surfaces and hydrodynamic boundary conditions, Langmuir 24, 11533 (2008).

[40] A. Maali, R. Boisgard, H. Chraibi, Z. Zhang, H. Kellay, and A. Würger, Viscoelastic Drag Forces and Crossover from No-Slip to Slip Boundary Conditions for Flow Near Air-Water Interfaces, Phys. Rev. Lett. 118, 084501 (2017).

[41] Y. Wang, B. Zeng, H. T. Alem, Z. Zhang, E. Charlaix, and A. Maali, Viscocapillary response of gas bubbles probed by thermal noise atomic force measurement, Langmuir 34, 1371 (2018).

[42] A. Steinberger, C. Cottin-Bizonne, P. Kleimann, and E. Charlaix, High friction on a bubble mattress, Nat. Mater. 6, 665 (2007).

[43] S. Leroy and E. Charlaix, Hydrodynamic interactions for the measurement of thin film elastic properties, J. Fluid Mech. 674, 389 (2011).

[44] S. Leroy, A. Steinberger, C. Cottin-Bizonne, F. Restagno, L. Léger, and É. Charlaix, Hydrodynamic Interaction Be- tween a Spherical Particle and an Elastic Surface: A Gentle Probe for Soft Thin Films, Phys. Rev. Lett. 108, 264501 (2012).

[45] R. Villey, E. Martinot, C. Cottin-Bizonne, M. Phaner-Goutorbe, L. Léger, F. Restagno, and E. Charlaix, Effect of Surface Elasticity on the Rheology of Nanometric Liquids, Phys. Rev. Lett. 111, 215701 (2013).

[46] D. Guan, C. Barraud, E. Charlaix, and P. Tong, Noncontact viscoelastic measurement of polymer thin films in a liquid medium using long-needle atomic force microscopy, Langmuir 33, 1385 (2017).

[47] D. Guan, E. Charlaix, R. Z. Qi, and P. Tong, Noncontact Viscoelastic Imaging of Living Cells Using a Long-Needle Atomic Force Microscope with Dual-Frequency Modulation, Phys. Rev. Appl. 8, 044010 (2017).

[48] Z. Zhang, V. Bertin, M. Arshad, E. Raphaël, T. Salez, and A. Maali, Direct Measurement of the Elastohydrodynamic Lift Force at the Nanoscale, Phys. Rev. Lett. 124, 054502 (2020).

[49] A. Maali and R. Boisgard, Precise damping and stiffness extraction in acoustic driven cantilever in liquid, J. Appl. Phys. 114, 144302 (2013).

[50] O. Reynolds, IV. On the theory of lubrication and its application to Mr. Beauchamp tower's experiments, including an experimental determination of the viscosity of olive oil, Philos. Trans. R. Soc. London 177, 157 (1886).

[51] See Supplemental Material at http://link.aps.org/supplemental/ 10.1103/PhysRevResearch.3.L032007 for further technical details on the theoretical model and experiments.

[52] V. S. Craig and C. Neto, In situ calibration of colloid probe cantilevers in force microscopy: hydrodynamic drag on a sphere approaching a wall, Langmuir 17, 6018 (2001).

[53] J. M. Skotheim and L. Mahadevan, Soft lubrication: The elastohydrodynamics of nonconforming and conforming contacts, Phys. Fluids 17, 092101 (2005).

[54] B. Saintyves, T. Jules, T. Salez, and L. Mahadevan, Selfsustained lift and low friction via soft lubrication, Proc. Natl. Acad. Sci. (USA) 113, 5847 (2016).

[55] Y. Moroi, K. Motomura, and R. Matuura, The critical micelle concentration of sodium dodecyl sulfate-bivalent metal dodecyl sulfate mixtures in aqueous solutions, J. Colloid Interface Sci. 46, 111 (1974).

[56] E. Fuguet, C. Ràfols, M. Rosés, and E. Bosch, Critical micelle concentration of surfactants in aqueous buffered and unbuffered systems, Anal. Chim. Acta 548, 95 (2005).

[57] K. Joshi and J. F. Gilchrist, Effect of added surfactant on convective assembly of monosized microspheres, Appl. Phys. Lett. 116, 083702 (2020). 